\title{
On Optimal Operator for Combining Left and Right Sole Pressure Data in Biometrics Security
}

\author{
Takahiro Takeda, ${ }^{1}$ Kei Kuramoto, ${ }^{1,2}$ Syoji Kobashi, ${ }^{1,2}$ and Yutaka Hata ${ }^{1,2}$ \\ ${ }^{1}$ Graduate School of Engineering, University of Hyogo, 2167 Shosha, Himeji, Hyogo 671-2280, Japan \\ ${ }^{2}$ WPI Immunology Frontier Research Center, Osaka University, 3-1 Yamada-Oka, Suita, Osaka 565-0871, Japan \\ Correspondence should be addressed to Takahiro Takeda; takahiro_takeda@ieee.org
}

Received 28 June 2013; Accepted 22 October 2013

Academic Editor: Ning Xiong

Copyright (c) 2013 Takahiro Takeda et al. This is an open access article distributed under the Creative Commons Attribution License, which permits unrestricted use, distribution, and reproduction in any medium, provided the original work is properly cited.

\begin{abstract}
This paper describes optimal operator for combining left and right sole pressure data in a personal authentication method by dynamic change of sole pressure distribution while walking. The method employs a pair of right and left sole pressure distribution change data. These data are acquired by a mat-type load distribution sensor. The system extracts features based on shape of sole and weight shift from each sole pressure distribution. We calculate fuzzy degrees of right and left sole pressures for a registered person. Fuzzy if-then rules for each registered person are statistically determined by learning data set. Next, we combine the fuzzy degrees of right and left sole pressure data. In this process, we consider six combination operators. We examine which operator achieves best accuracy for the personal authentication. In the authentication system, we identify the walking persons as a registered person with the highest fuzzy degree. We verify the walking person as the target person when the combined fuzzy degree of the walking person is higher than a threshold. In our experiment, we employed 90 volunteers, and our method obtained higher authentication performance by mean and weighted sum operators.
\end{abstract}

\section{Introduction}

Information technologies and network-based services, such as healthcare, commercial, and social services become indispensable parts of our lives. Reliable authentication of users is needed for secure access to these services to avoid compromising our privacy. Passwords and PINs are still the major authentication methods for network services. However, we have to remember a lot of passwords or PINs for several services. Moreover, the information might be stolen by shoulder surfing, keystroke logging and so on. Biometrics is an emerging technology to authenticate a person based on physical or behavioral features. While techniques using physical features such as fingerprint $[1,2]$ and iris can achieve high recognition accuracy, behavioral features such as signature [3], speech [4], and walking [5-10] are more user friendly.

We focus on a biometric method based on sole pressure and dynamics in walking. Because walking is the most natural daily motion, biometrics systems that use walking do not require any training for authentication. This method can be conveniently used to authenticate people while passing through a door or passageway. It is available to an application where a person entering a room and walking toward a device or a computer can be authenticated and immediately logged in the room. Walking patterns can be captured with cameras $[5,6]$, acceleration sensors [7], and pressure sensors [8-14]. The pressure sensor measures a walking as a dynamic change of pressures. Several studies [8-14] have been introduced on this topic. Addlesee et al. [10], Orr and Abowd [11], and Yamakawa et al. [12] focus on sole pressure change. These methods use some features such as peak pressure value, time to peak pressure, and form of pressure change curve. They authenticate the walking person by employing only one foot. Qian et al. [9] has used step lengths, 3D trajectories of center of pressure, and angles between right and left soles. They measure over three steps for authentication by the use of large pressure sensor $(3.7 \mathrm{~m} \times 4.5 \mathrm{~m})$. However, these methods did not use shape information of the sole. Jung et al. [8] use footprint and center-of-pressure trajectory 
for authentication. They employ both of right and left sole pressures, but their pressure sensor is too small to measure both feet $(40 \mathrm{~cm} \times 80 \mathrm{~cm})$. Furthermore, authentication accuracies of these methods are not enough to use them for security field.

In this study, we propose a kind of integration approach [15-17] to improve authentication performance of biometrics based on walking. Integration approaches improve authentication accuracy by combining multimethods, multifeatures, or multisampling. The proposed method combines the authentication score of right and left sole pressure data. In our previous study, we have proposed a personal authentication method by using only right sole pressure [13]. This study employed fuzzy degree calculated from sole pressure change of one sole as an authentication score. Moreover, in [14] we combined authentication scores of right and left sole by mean operator. However, we did not compare the mean operator with other operators. Thus, this paper discusses an optical operator for combining left and right sole pressure data in fuzzy logic approached biometrics security. These right and left sole pressures are measured by middle size load distribution sensor, and it is designed to accommodate two steps of an average walker. The load distribution sensor is a kind of pressure sensors arranged on a two-dimensional pattern. This arrangement of sensors enables us to acquire dynamic change of sole pressure distribution. To authenticate person, gait features are extracted from the sole pressure distribution. The gait features consist both of sole pressure change and footprint. As the authentication score, the system calculates fuzzy degrees of each sole pressure for registered person. The fuzzy degree means a degree of similarity of acquired sole pressure and registered data set for a person. Here, fuzzy membership functions are statistically determined in learning process. The system combines the fuzzy degree of right and left sole by a combination operator and uses it for authentication. In the experiment, we employed six combination operators and evaluated these authentication performances. We show the experimental results of 90 volunteers of each combination operator. Then, we describe an optimal operator for combining left and right sole pressures.

This paper is organized as follows. Section 2 presents the setup and data set. Section 3 introduces features extraction. Section 4 shows calculation of fuzzy degree for one sole. Section 5 introduces personal authentication by combined fuzzy degree. Section 6 shows experimental results. Finally, Sections 7 and 8 discuss and conclude our study.

\section{Preliminaries}

2.1. Load Distribution Sensor. In our system, a sole pressure data while walking is acquired by a mat-type load distribution sensor (Arrow Industry Co., Ltd. AS-64X256-7PM). The load distribution sensor is a kind of pressure sensor. Figure 1 shows overview of our data acquisition system. As shown in this figure, sensing area of the sensor is $33 \mathrm{~cm}$ (width) $\times$ $176 \mathrm{~cm}$ (length), and it is enough to acquire sole pressures of left and right soles. Our experimental system consists of a load distribution sensor, a control device (Arrow Industry Co., Ltd. AS-64X256), and a personal computer.

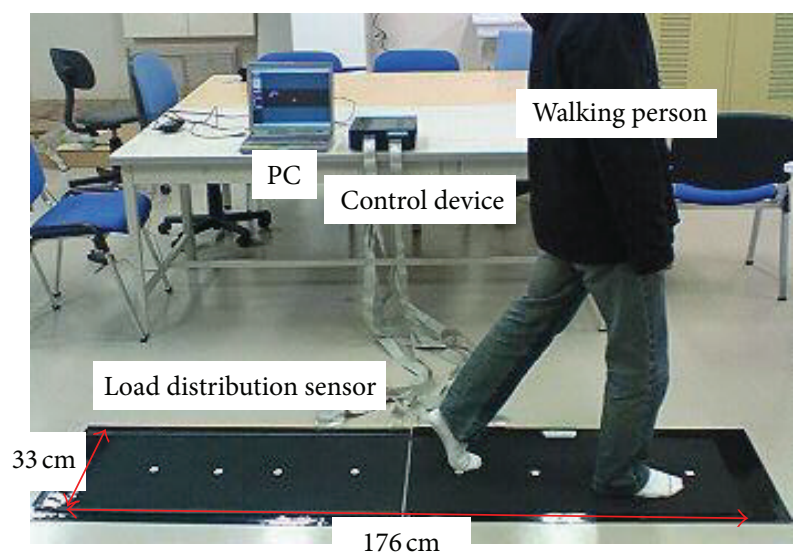

FIGURE 1: Overview of the data acquisition system.

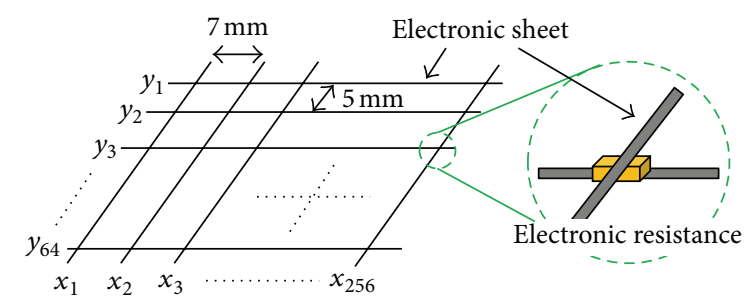

Figure 2: Internal constitution of the load distribution sensor.

Figure 2 illustrates internal constitution of the load distribution sensor. As shown in this figure, the sensor has 64 vertical electrode sheets and 256 transverse electrode sheets. Distance between the nearest electrode sheets is $5.0 \mathrm{~mm}$ in a vertical direction ( $y$-axis) and $7.0 \mathrm{~mm}$ in transverse direction $(x$ axis). In every intersection of vertical and transverse sheets, an electric resistance element is sandwiched in two electrode sheets and this intersection point is a sensing point. Then, the load distribution sensor has $256 \times 64$ sensing points. When we tread the sensing points, the corresponding elements decrease electric resistance. The control device converts this electric resistance into 8 bit (256 level) digital value and provides all the values to a personal computer. The personal computer stores the pressure value at the sampling interval $100 \mathrm{~ms}$. Figure 3 shows a load distribution data of one sampling time. In this paper, we show the pressure distribution data as 8 bit grayscale image. High pressure points are colored with black and low pressure points are done with white.

2.2. Data Acquisition. In the experiments, subjects were asked to walk normally on the load distribution sensor platform. The protocol is such that the subject must first halt right before the sensing area and then walk through and past the sensor, typically involving two or three steps. We consider the walking sense as the $x$-axis of the sensor. The system acquires the sole pressure distribution during these steps and stores data in a computer as biometric evidence. One limitation of the experiment is that the subjects must walk with bare feet or with socks. Figure 4 shows an example of spatiotemporal sole pressure data while walking. 


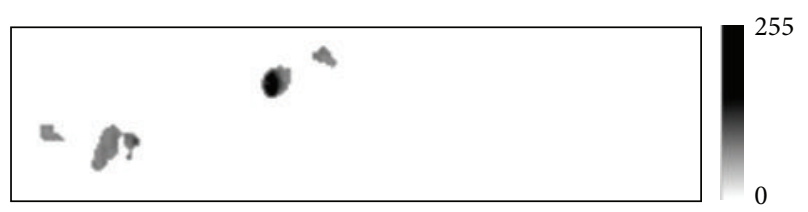

FIgURE 3: An example of load distribution data of one sampling time.

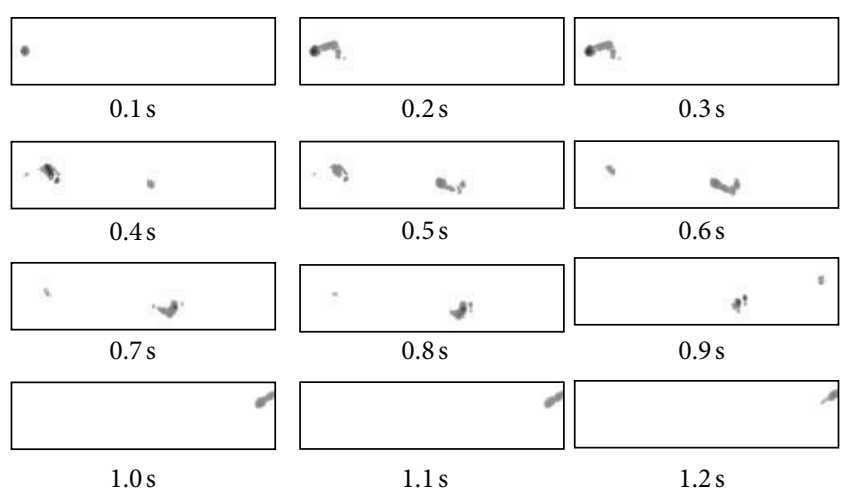

Figure 4: Examples of load distribution while walking.

2.3. Data Set. From the acquired sole pressure data, the system separates a right sole pressure data and a left sole pressure data. We define sole coordinate system as shown in Figure 5. We consider a registered person set $Y=\left\{y_{1}, y_{2}\right.$, $\left.\ldots, y_{r}, \ldots, y_{n}\right\}$. Here, the notation $n$ denotes the number of registered person. We take the sole pressure distribution data $N$ times from each registered person and use these pressure data in learning process of our system. The notation $X^{S}$ denotes a pressure distribution change data when a person " $y_{s}$ " is walking. A pressure data $X^{S}$ has a right sole pressure data $X_{R}^{S}$ and a left sole pressure data $X_{L}^{S}$.

\section{Features Extraction}

For authentication, our system extracts thirty-nine gait features from each sole pressure data $X_{k}^{S}$, where the notation $k$ denotes the index of the right foot $(k=R)$ or the left foot $(k=$ $L)$. Twelve features are based on a footprint, and twenty-seven features are based on weight shift while walking. The notation $f_{i}\left(X_{k}^{S}\right)$ denotes a gait feature with respect to a sole pressure data $X_{k}^{S}$, where $i$ denotes the index of each feature. Table 1 shows the notation indexes with respect to a sole pressure data $X_{k}^{S}$.

The system extracts twelve features $f_{i}\left(X_{k}^{S}\right)$ from every footprint made from a sole pressure data $X_{k}^{S}$. A footprint is one of overlapped pressure distribution of the sole, and it is obtained by maximum of binary images of pressure distribution for all frames. Figure 6 shows procedure of the footprint production. Binary images are calculated from every frame of the sole pressure data. Black pixels have pressure values and white pixels do not have pressure value. From this footprint, we extract the length of footprint $f_{1}\left(X_{k}^{S}\right)$, the width of footprint $f_{2}\left(X_{k}^{S}\right)$, the area of footprint $f_{3}\left(X_{k}^{S}\right)$, the angel between the direction of footprint and $x$-axis of load distribution sensor $f_{4}\left(X_{k}^{S}\right)$, and the distribution of footprint $f_{5}\left(X_{k}^{S}\right), \ldots, f_{12}\left(X_{k}^{S}\right)$ as features. It is shown in Figure 7. In this figure, white colored area shows nonsole area and black colored areas show the footprint. The area of footprint $f_{3}\left(X_{k}^{S}\right)$ is calculated by the number of sole pixels in the footprint. The area of footprint shows a sole size of the walking person. The red straight line is obtained by least square method for footprint. We calculate the angle $f_{4}\left(X_{k}^{S}\right)$ between the red line and $x$-axis of the sensor. This feature means an angle between a foot and walking direction because the data acquisition protocol sets the $x$-axis as a direction of walking. The green rectangle is the circumscribed rectangle. We use the length of long side of the this green circumscribed rectangle as the length of footprint $f_{1}\left(X_{k}^{S}\right)$ and use the length of short side as the width of footprint $f_{2}\left(X_{k}^{S}\right)$ as the features. Since there are differences in the length and width of foot between individuals, we employ these features for authentication. The green dotted lines are quarter of the green circumscribed rectangle. Then, we obtain eight small areas surrounded by the red straight line, the green circumscribed rectangle and the green dotted lines. The distribution of footprint $f_{5}\left(X_{k}^{S}\right)$, $\ldots, f_{12}\left(X_{k}^{S}\right)$ are calculated by number of sole pixels in each small areas. For example, when the walking person has a flat foot, the area of arch on footprint $f_{6}\left(X_{k}^{S}\right)$ and $f_{7}\left(X_{k}^{S}\right)$ is bigger than that of normal foot. Moreover, several footprints lack the area of toes $f_{12}\left(X_{k}^{S}\right)$, when the walker puts his/her weight on only a big toe. As seen from above, the area of footprint $f_{3}\left(X_{k}^{S}\right)$ shows a size of footprint, whereas the distribution of footprint $f_{5}\left(X_{k}^{S}\right), \ldots, f_{12}\left(X_{k}^{S}\right)$ express foot shape and posture of walking.

The system extracts twenty-seven features from every frame of a sole pressure data $X_{k}^{S}$. We calculate the center of sole pressure (CSP), the highest sole pressure point (HSP), and the area of footprint from each frame. The area is calculated by the number of sole pixels from each frame. Figure 8 shows examples of CSPs and HSPs of each frame. Generally, the area changes with a period of gait cycle, and the changes express weight shift of the walker. The CSPs and HSPs show significant locations of sole pressure at the time. Generally, these trajectories are drawn lines from heel to toe. The CSPs trajectory is famous feature of gait analysis, and it is related to center-of-gravity while walking. On the other hand, the HSP shows a point that is applied to the highest power by the walker at the time. Figure 9 shows two different right sole pressure data. Thus, these two data vary in the number of frames; thus, the gait periods of two sole pressures are different. To solve this problem, we normalize these features to nine frames sole data. We suppose that the dynamic changes of each feature are similar in one step. Therefore, the system approximates these features by a polygonal line, and the nine features are interpolated by liner interpolation. The interpolated nine CSPs $f_{13}\left(X_{k}^{S}\right), \ldots, f_{21}\left(X_{k}^{S}\right)$ and nine HSPs $f_{22}\left(X_{k}^{S}\right), \ldots, f_{30}\left(X_{k}^{S}\right)$ are extracted from these trajectory, as shown in Figure 10. The interpolated nine areas 


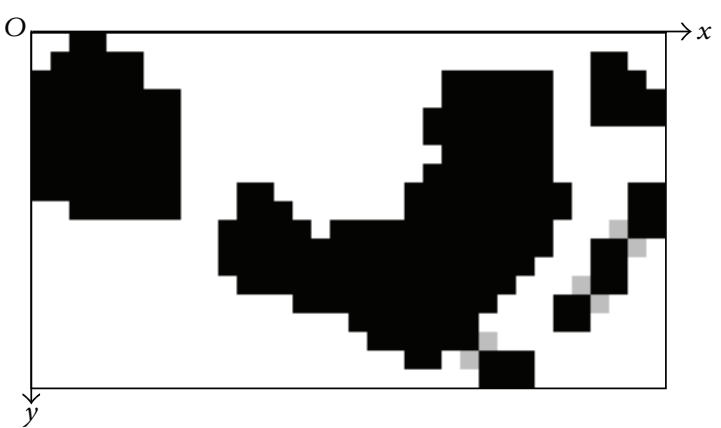

(a) Right sole

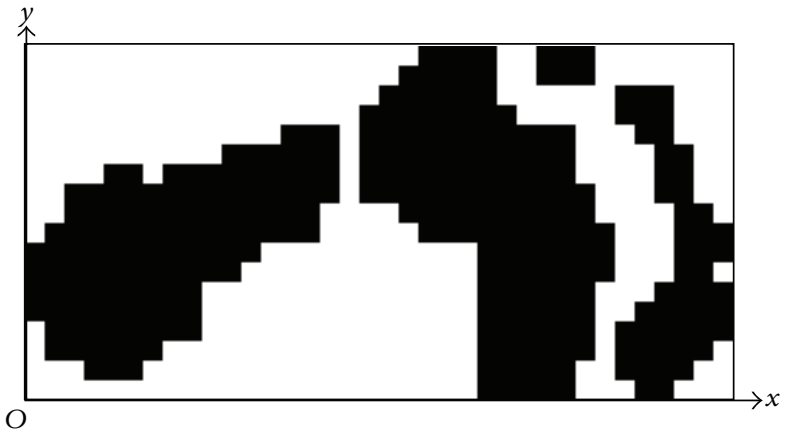

(b) Left sole

FIgURE 5: Right and left sole coordinate systems.

TABLE 1: Features of each sole pressure data.

\begin{tabular}{ll}
\hline Notation & $\begin{array}{c}\text { Sole pressure data } X_{k}^{S} \\
\text { Feature }\end{array}$ \\
\hline$f_{1}\left(X_{k}^{S}\right)$ & The length of footprint \\
$f_{2}\left(X_{k}^{S}\right)$ & The width of footprint \\
$f_{3}\left(X_{k}^{S}\right)$ & The area of footprint \\
$f_{4}\left(X_{k}^{S}\right)$ & The angle of footprint \\
$f_{5}\left(X_{k}^{S}\right)$ & The area of inner side of heel \\
$f_{6}\left(X_{k}^{S}\right), f_{7}\left(X_{k}^{S}\right)$ & The area of arch on footprint \\
$f_{8}\left(X_{k}^{S}\right)$ & The area of a big toe on footprint \\
$f_{9}\left(X_{k}^{S}\right)$ & The area of outer side of heel on footprint \\
$f_{10}\left(X_{k}^{S}\right), f_{11}\left(X_{k}^{S}\right)$ & The area of arch on footprint \\
$f_{12}\left(X_{k}^{S}\right)$ & The area of toes on footprint \\
$f_{13}\left(X_{k}^{S}\right) \sim f_{21}\left(X_{k}^{S}\right)$ & The normalized CSPs on footprint \\
$f_{22}\left(X_{k}^{S}\right) \sim f_{30}\left(X_{k}^{S}\right)$ & The normalized HSPs on footprint \\
$f_{31}\left(X_{k}^{S}\right) \sim f_{39}\left(X_{k}^{S}\right)$ & The normalized areas on footprint \\
\hline
\end{tabular}

$f_{31}\left(X_{k}^{S}\right), \ldots, f_{39}\left(X_{k}^{S}\right)$ are extracted from the polygonal lines as shown in Figure 11.

\section{Fuzzy Degree for One Sole}

In this section, we explain a calculation method of two fuzzy degrees $\mu_{R}^{r}\left(X_{R}^{S}\right)$ and $\mu_{L}^{r}\left(X_{L}^{S}\right)$ for a registered person $y_{r}$. The fuzzy degrees $\mu_{R}^{r}\left(X_{R}^{S}\right)$ and $\mu_{L}^{r}\left(X_{L}^{S}\right)$ are calculated from each sole pressure data $X_{R}^{S}$ and $X_{L}^{S}$, respectively. We employ these fuzzy degrees for authentication score of each foot. We consider the following as knowledge about gait features for biometrics security.

Knowledge 1. Features of same person are similar.

Knowledge 2. Feature with higher classification score in learning pressure is more primary for personal authentication than for other features.

From this knowledge, the following fuzzy if-then rules are derived.

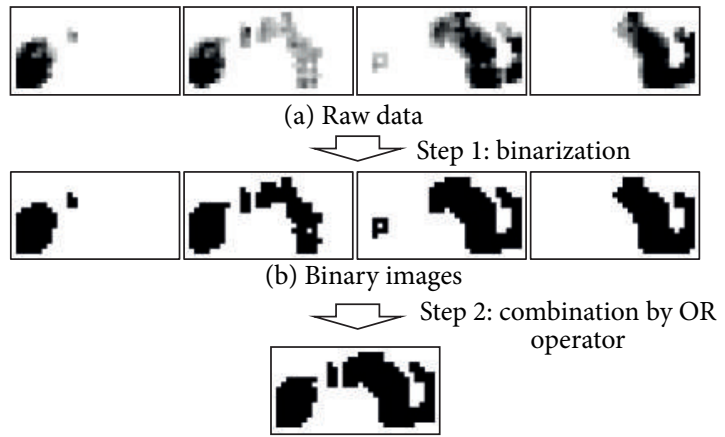

(c) Footprint (left sole)

FIgURE 6: Procedure of the footprint production.

Rule 1. If feature $f_{i}\left(X_{k}^{S}\right)$ is $\operatorname{CLOSE}_{k, i}$ to a baseline value $\alpha_{k, i}^{r}$ of a registered person $y_{r}$, then the degree of similarity $P_{k, i}^{r}\left(X_{k}^{S}\right)$ of the feature is high.

Rule 2. If classification score $\beta_{k, i}^{r}$ of learning sole pressure data is $\mathrm{HIGH}_{k, i}$, then the degree of contribution $W_{k, i}^{r}\left(\beta_{k, i}^{r}\right)$ for the authentication of the feature is high.

The fuzzy membership functions $\mathrm{CLOSE}_{k, i}$ and $\mathrm{HIGH}_{k, i}$ are defined in Figure 12. These functions have parameters $\alpha_{k, i}^{r}, t_{k, i}^{r}, u_{k, i}^{r}, \beta_{k, i}^{r}$, and $\operatorname{sum}_{k}^{r}$. Here, the notations $t_{k, i}^{r}$ and $u_{k, i}^{r}$ denote width of the membership function CLOSE CL,i . The notation $\operatorname{sum}_{k}^{r}$ is a sum of the classification score $\beta_{k, i}^{r}$. These parameters are determined by learning process. The determination method of these parameters for any $k$ and $i$ is as follows. Consider one test data and $N$ learning data for a person, and determine the parameters by the learning data of all registered persons for the test data. First, the system calculates the mean $\operatorname{Mean}_{k, i}^{r}$ and a standard deviation $\operatorname{SD}_{k, i}^{r}$ of $N$ learning data for $k$ and $i$ of $y_{r}$. Second, we initialize $\alpha_{k, i}^{r, i} \leftarrow$ $\operatorname{Mean}_{k, i}^{r}, t_{k, i}^{r} \leftarrow \mathrm{SD}_{k, i}^{r}$, and $u_{k, i}^{r} \leftarrow \mathrm{SD}_{k, i}^{r}$. By this initialization, the membership function, CLOSE $E_{k, i}$, is formed as shown in Figure 13(a), and classification score $\beta_{k, i}^{r}$ is calculated by the following equation:

$$
\beta_{k, i}^{r}=\frac{\sum_{j=1}^{N} P_{k, i}^{r}\left(L_{k}^{r, j}\right)}{N}-\frac{\sum_{j=1}^{N \times(n-1)} P_{k, i}^{r}\left(L_{k}^{t, j}\right)}{N \times(n-1)} .
$$




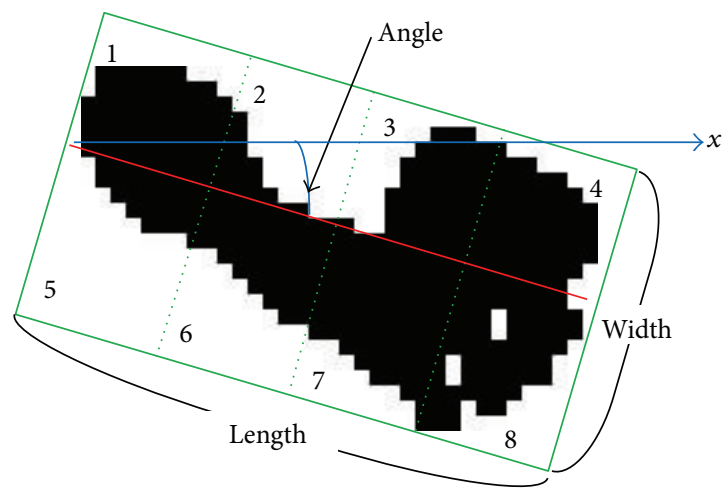

(a) Right sole

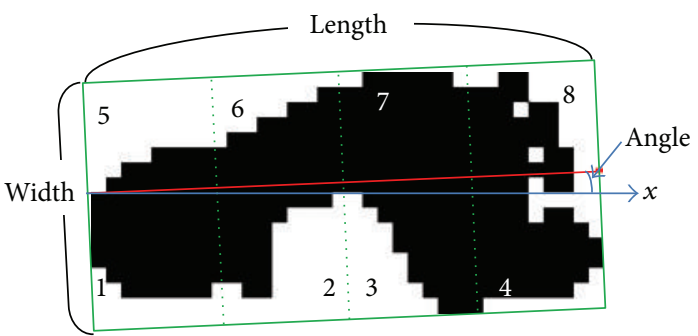

(b) Left sole

Figure 7: Example of footprint and these features.

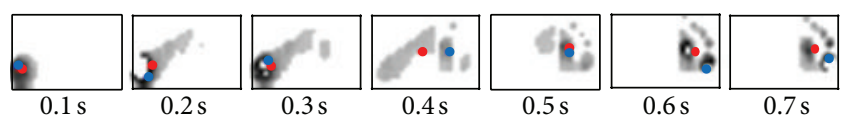

- CSP

- HSP

FIGURE 8: Example of CSP and HSP of each frame.

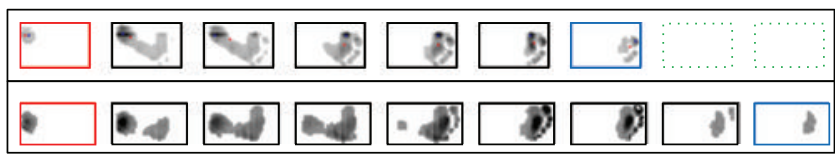

Initial contact

Toe off

FIGURE 9: Two sole data that show the number of frames is different.

Here, the notation $L_{k}^{r, j}$ and $L_{k}^{t, j}$ denote learning data of the target person $y_{r}$ and those of other registered persons, respectively. The notation $j$ denotes an index of learning data. The notation $N$ denotes the number of training samples of a registered person, and the notation $n$ denotes the number of registered person. The fuzzy degree of similarity $P_{k, i}^{r}\left(X_{k}^{S}\right)$ of the feature is calculated by the following equation:

$$
P_{k, i}^{r}\left(X_{k}\right)=\min \left(\operatorname{CLOSE}, S_{f_{i}\left(X_{k}\right)}(f)\right) .
$$

Here, we define fuzzy singleton function $S_{a}(b)$ by the following equation:

$$
S_{a}(b)= \begin{cases}1, & \text { if } b=a \\ 0, & \text { otherwise }\end{cases}
$$

Equation (1) means the difference between the average degree of similarity $P_{k, i}^{r}\left(L_{k}^{t}\right)$ for other person and the average $P_{k, i}^{r}\left(L_{k}^{r}\right)$ for the target person $y_{r}$. We employ this value as the classification score. Third, the system repeats the calculation of (1) for all domains of $\mathrm{Mean}_{k, i}^{r}-\mathrm{SD}_{k, i}^{r} \leq \alpha_{k, i}^{r} \leq$ $\operatorname{Mean}_{k, i}^{r}+\mathrm{SD}_{k, i}^{r}, 0 \leq t_{k, i}^{r} \leq 4 \mathrm{SD}_{k, i}^{r}$ and $0 \leq u_{k, i}^{r} \leq 4 \mathrm{SD}_{k, i}^{r}$ at the interval of $\mathrm{SD}_{k, i}^{r} / 10$. Figure 13(b) shows examples of the deformed membership function $\mathrm{CLOSE}_{k, i}$. We obtain

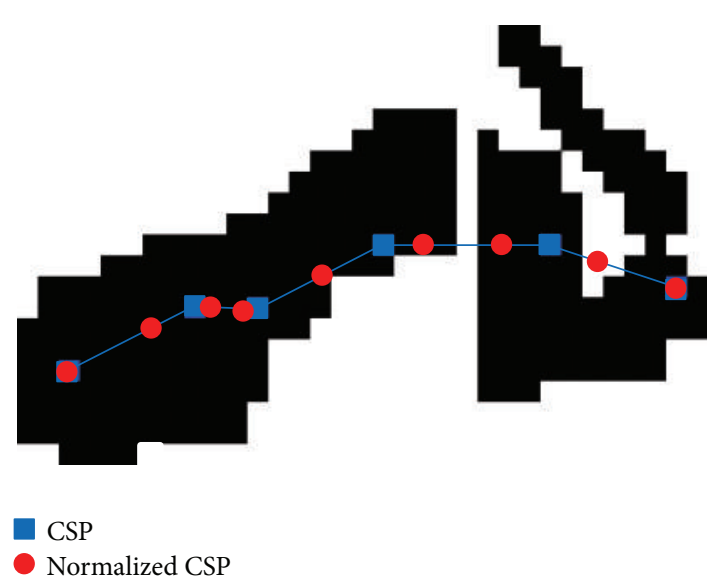

FIGURE 10: An example of CSPs trajectory and nine normalized CSPs.

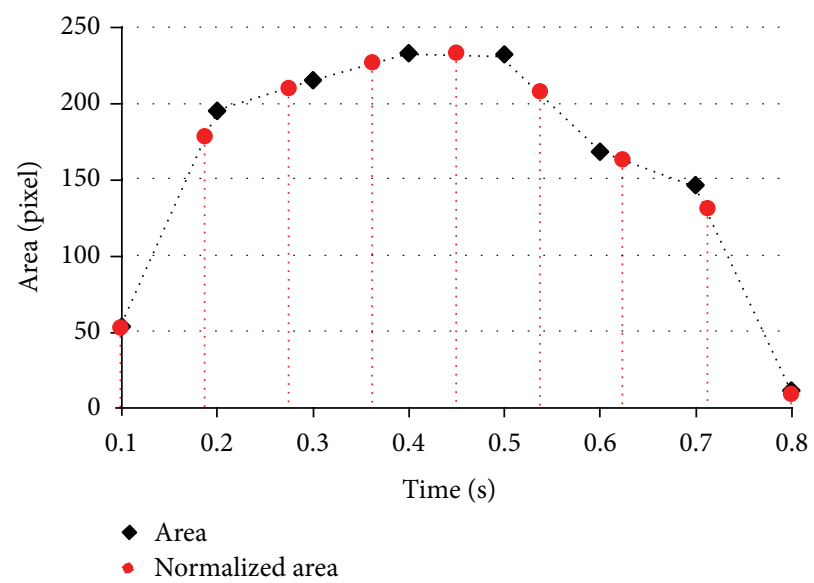

FIGURE 11: Example of nine normalize areas.

the classification score $\beta_{k, i}^{r}$ from all domains. The system employs these parameters $\alpha_{k, i}^{r}, t_{k, i}^{r}$, and $u_{k, i}^{r}$ with the highest $\beta_{k, i}^{r}$ among all domains for a feature $f_{i}\left(X_{k}^{r}\right)$ of person $y_{r}$. The determination process are repeated for all $k$ and $i$ of $y_{r}$. 


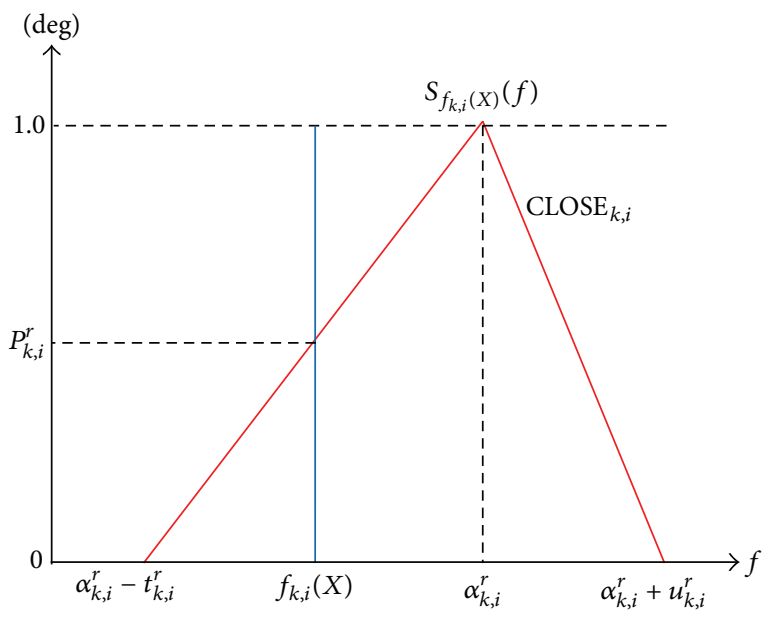

(a) Rule 1 (CLOSE)

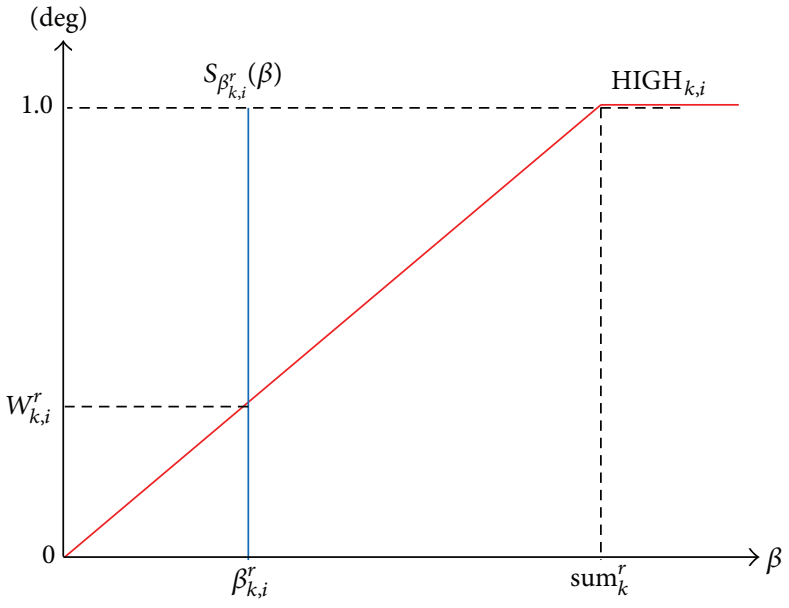

(b) Rule 2 (HIGH)

FIGURE 12: Fuzzy membership functions.

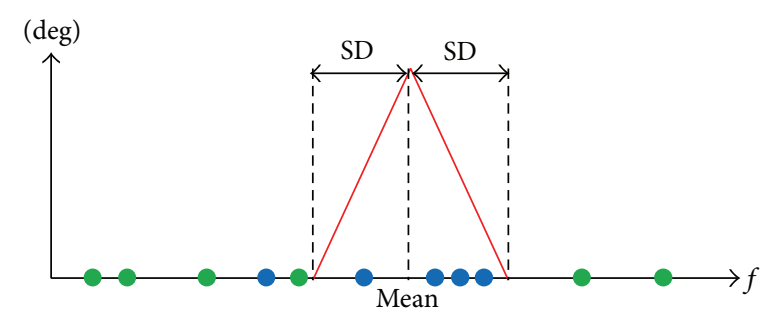

- $L_{k}^{r}$

$L_{k}^{t}$

(a) Example of initialized fuzzy membership function CLOSE
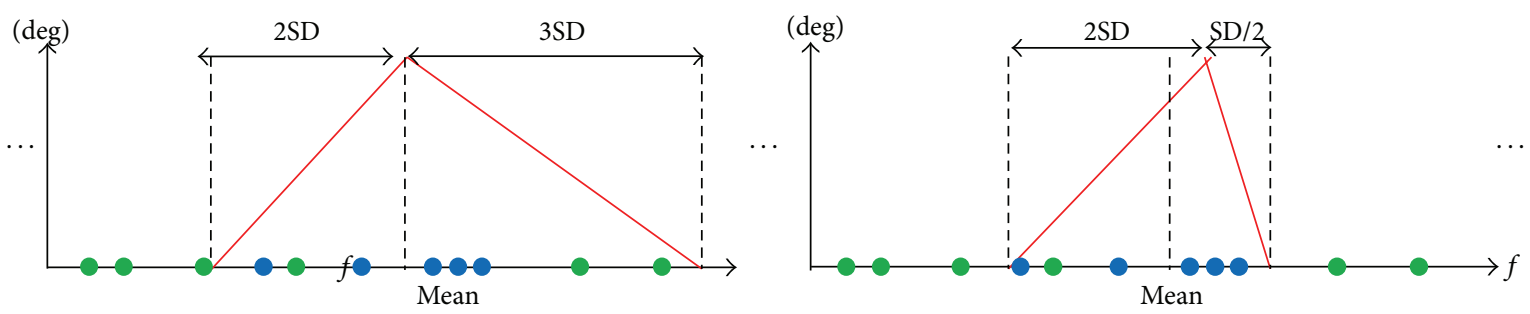

- $L_{k}^{r}$

(b) Example of deformed fuzzy membership function CLOSE

FIGURE 13: Examples of fuzzy membership functions deformation.

The parameter $\operatorname{sum}_{k}^{r}$ of membership function $\mathrm{HIGH}_{k, i}$ is calculated by sum of classification score $\beta_{k, i}^{r}$ of a person $y_{r}$. It is calculated by the following equation:

$$
\operatorname{sum}_{k}^{r}=\sum_{i=1}^{39} \beta_{k, i}^{r} .
$$

The fuzzy degree for a feature $Q_{k, i}^{r}\left(X_{k}^{S}\right)$ is defined by (5). We calculate the degree $Q_{k, i}^{r}\left(X_{k}^{S}\right)$ for all $k$ and $i$ of pressure data $X_{k}^{S}$ as

$$
Q_{k, i}^{r}\left(X_{k}^{S}\right)=P_{k, i}^{r}\left(X_{k}^{S}\right) \times W_{k, i}^{r}\left(\beta_{k, i}^{r}\right) .
$$

Here the fuzzy degree of contribution $W_{k, i}^{r}\left(\beta_{k, i}^{r}\right)$ means the effectiveness of $f_{i}\left(X_{k}^{S}\right)$ for person $y_{r}$, and it is defined by the following equation:

$$
W_{k, i}^{r}\left(\beta_{k, i}^{r}\right)=\min \left(\mathrm{HIGH}_{k, i}, S_{\beta_{k, i}^{r}}(w)\right)
$$

Finally, the system calculates a fuzzy degree $\mu_{k}^{r}\left(X_{k}^{S}\right)$ of a sole pressure data $X_{k}^{S}$ for all $y_{r}$ by (7). Here, the fuzzy degree of a sole pressure $\mu_{k}^{r}\left(X_{k}^{S}\right)$ means an authentication score for 
the registered person calculated from one sole pressure data $X_{k}^{S}$ as

$$
\mu_{k}^{r}\left(X_{k}^{S}\right)=\sum_{i=1}^{39} Q_{k, i}^{r}\left(X_{k}^{S}\right)
$$

\section{Fuzzy Degree for a Walking Data}

In order to authenticate a person, we calculate a combined fuzzy degree $\mu_{\text {sole }}^{r}\left(X^{S}\right)$. The combined fuzzy degree is calculated from fuzzy degrees of right sole $\mu_{R}^{r}\left(X_{R}^{S}\right)$ and left sole $\mu_{L}^{r}\left(X_{L}^{S}\right)$. In this method, we identify and verify person by the fuzzy degree $\mu_{\text {sole }}^{r}\left(X^{S}\right)$. For personal identification $(1: N$ collation), the system calculates the combined fuzzy degree $\mu_{\text {sole }}^{r}\left(X^{S}\right)$ for every registered person $y_{s}$. We obtain the highest fuzzy degree among the registered persons. The system identifies the walking person $y_{s}$ as a registered person $y_{r}$ with the highest combined fuzzy degree. For personal verification ( $1: 1$ collation), the system calculates combined fuzzy degree of $X^{S}$ for a target person $y_{r}$. If the combined fuzzy degree is higher than a threshed, the system verifies the walking person $y_{s}$ as the target person $y_{r}$.

In this paper, we employ mean (8), minimum (9), maximum (10), product, probabilistic sum (11), and weighted sum (12) as the combination operator. And, we research an optimal combination operator for our biometrics authentication. In these six operators, the fuzzy degree $\mu_{\text {sole }}^{r}\left(X^{S}\right)$ is always in the real interval $[0,1]$ because $\mu_{R}^{r}\left(X_{R}^{S}\right)$ and $\mu_{L}^{r}\left(X_{L}^{S}\right)$ are within $[0,1]$

$$
\begin{gathered}
\mu_{\text {Sole }}^{r}\left(X^{S}\right)=\frac{1}{2}\left\{\mu_{R}^{r}\left(X_{R}^{S}\right)+\mu_{L}^{r}\left(X_{L}^{S}\right)\right\}, \\
\mu_{\text {Sole }}^{r}\left(X^{S}\right)= \begin{cases}\mu_{R}^{r}\left(X_{R}^{S}\right), & \text { if } \mu_{R}^{r}\left(X_{R}^{S}\right)<\mu_{L}^{r}\left(X_{L}^{S}\right), \\
\mu_{L}^{r}\left(X_{L}^{S}\right), & \text { otherwise, }\end{cases} \\
\mu_{\text {Sole }}^{r}\left(X^{S}\right)= \begin{cases}\mu_{R}^{r}\left(X_{R}^{S}\right), & \text { if } \mu_{R}^{r}\left(X_{R}^{S}\right)>\mu_{L}^{r}\left(X_{L}^{S}\right), \\
\mu_{L}^{r}\left(X_{L}^{S}\right), & \text { otherwise, }\end{cases} \\
\mu_{\text {Sole }}^{r}\left(X^{S}\right)=\left\{\mu_{R}^{r}\left(X_{R}^{S}\right)+\mu_{L}^{r}\left(X_{L}^{S}\right)\right\}-\left\{\mu_{R}^{r}\left(X_{R}^{S}\right) \times \mu_{L}^{r}\left(X_{L}^{S}\right)\right\}, \\
\mu_{\text {Sole }}^{r}\left(X^{S}\right)=w \times \mu_{R}^{r}\left(X_{R}^{S}\right)+(1-w) \times \mu_{L}^{r}\left(X_{L}^{S}\right) .
\end{gathered}
$$

Here, the notation $w$ is the weighting parameter, and $w$ is within $[0,1]$. When $w$ is 0 , the combined fuzzy degree equals the fuzzy degree of the left sole pressure, and when $w$ is 1.0, the combined fuzzy degree equals the fuzzy degree of the right sole pressure. In addition, when $w$ is 0.5 , the weighting sum operator means the mean operator.

\section{Experimental Results}

In this experiment, we employed 90 volunteers as shown in Table 2. For each volunteer, we took the sole pressure data six times. These sole pressure data are acquired with socks. We used five data for learning and used one data as test data. We evaluate the proposed method by sixfold cross-validation
TABLE 2: Volunteer's age and gender information.

\begin{tabular}{lccc}
\hline Age (year) & Male (person) & Female (person) & Total (person) \\
\hline $20-29$ & 11 & 28 & 39 \\
$30-39$ & 4 & 3 & 7 \\
$40-49$ & 5 & 4 & 9 \\
$50-59$ & 2 & 4 & 6 \\
$60-69$ & 2 & 1 & 3 \\
$70-79$ & 8 & 9 & 17 \\
$80-89$ & 4 & 5 & 9 \\
\hline Total & 36 & 54 & 90 \\
\hline
\end{tabular}

method. False rejection rate (FRR), false acceptance rate (FAR), and equal error rate (EER) are employed for the performance test. FRR and FAR are defined by (13) and (14), respectively. FRR is concerned with the number of instance defined as an authorized individual being falsely rejected by an identification system. FAR is concerned with the number of instances defined as an unauthorized individual being falsely accepted by an identification system. The higher FRR decreases the user friendliness and higher FAR increases the risk of intrusion.

$$
\begin{gathered}
\mathrm{FRR}=\frac{\text { Number of false rejections }}{\text { Number of authorized attempts }} \times 100(\%), \\
\mathrm{FAR}=\frac{\text { Number of false acceptances }}{\text { Number of impostor attempts }} \times 100(\%) .
\end{gathered}
$$

The EER is the error rate that FRR, equal to FAR in verification. The lower EER implies higher accurate and more reliable personal authentication.

We identified and verified the subjects by the proposed method. We compared the combined fuzzy degrees $\mu_{\text {Sole }}^{r}\left(X^{S}\right)$ with the fuzzy degree of right sole $\mu_{R}^{r}\left(X_{R}^{S}\right)$ and left sole $\mu_{L}^{r}\left(X_{L}^{S}\right)$. In our past works, [13] authenticated person by only right sole pressure data. Reference [14] authenticated person by using mean value of right and left sole fuzzy degrees. Thus, $[13,14]$ correspond methods using the fuzzy degree of right sole $\mu_{R}^{r}\left(X_{R}^{S}\right)$ and mean operator, respectively. We compared authentication results of these combination operators with results of an authentication method based on Euclidean distance. In the authentication method based on Euclidean distance, firstly, the system normalizes every gait feature using the maximum and minimum value. Secondly, the system constructs template data for each registered person. The template data is made by mean value of learning data. Thirdly, the Euclidean distance $D^{r}$ is calculated by the following equation:

$$
D^{r}=\sqrt{\sum_{i=1}^{39}\left\{\operatorname{tmp}_{R, i}^{r}-f_{i}\left(X_{R}^{S}\right)\right\}^{2}+\sum_{i=1}^{39}\left\{\operatorname{tmp}_{L, i}^{r}-f_{i}\left(X_{L}^{S}\right)\right\}^{2}} .
$$

Here, $\operatorname{tmp}_{k}^{r}$ denotes the template data of the registered person $y_{r}$. In identification, the system calculates the Euclidean distance $D^{r}$ for every registered person, and the walking 
TABLE 3: Data set of performance test.

\begin{tabular}{lccccccc}
\hline Number of subjects & \multicolumn{5}{c}{ Age (year) } & (50-69 & $70-79$ \\
\hline 20 & $20-29$ & $30-39$ & $40-49$ & $50-59$ & 3 & 1 & 3 \\
30 & 10 & 1 & 1 & 3 & 1 & 5 \\
40 & 17 & 1 & 2 & 3 & 2 & 1 \\
50 & 18 & 3 & 5 & 3 & 3 & 1 \\
90 & 20 & 3 & 5 & 6 & 3 & 10 \\
\hline
\end{tabular}

TABLE 4: FRRs in identification of combination operators.

\begin{tabular}{|c|c|c|c|c|c|c|c|c|c|}
\hline \multirow{3}{*}{ Volunteers (person) } & \multicolumn{9}{|c|}{ FRR (\%) } \\
\hline & \multicolumn{6}{|c|}{ Combination operators } & \multirow[b]{2}{*}{$\mu_{R}^{r}(16)$} & \multirow[b]{2}{*}{$\mu_{L}^{r}$} & \multirow{2}{*}{ Euclidean distance } \\
\hline & Mean (17) & Minimum & Maximum & Product & Probabilistic & Weighting sum & & & \\
\hline 20 & 0.0 & 8.3 & 10.0 & 3.3 & 0.0 & $0.0(w=0.49)$ & 16.7 & 16.7 & 32.5 \\
\hline 30 & 3.9 & 14.4 & 13.9 & 5.6 & 4.4 & $3.3(w=0.51)$ & 22.8 & 27.2 & 45.6 \\
\hline 40 & 7.5 & 19.2 & 21.3 & 7.9 & 7.1 & $7.1(w=0.49)$ & 30.4 & 33.8 & 50.0 \\
\hline 50 & 12.7 & 27.7 & 26.3 & 13.3 & 13.3 & $11.7(w=0.52)$ & 40.0 & 41.7 & 56.3 \\
\hline 90 & 35.0 & 49.3 & 48.0 & 35.6 & 36.1 & $35.0(w=0.50)$ & 59.4 & 59.6 & 73.7 \\
\hline
\end{tabular}

TABLE 5: FARs in identification of combination operators.

\begin{tabular}{|c|c|c|c|c|c|c|c|c|c|}
\hline \multirow{3}{*}{ Volunteers (person) } & \multicolumn{9}{|c|}{ FAR (\%) } \\
\hline & \multicolumn{6}{|c|}{ Combination operators } & \multirow{2}{*}{$\mu_{R}^{r}(16)$} & \multirow{2}{*}{$\mu_{L}^{r}$} & \multirow{2}{*}{ Euclidean distance } \\
\hline & Mean (17) & Minimum & Maximum & Product & Probabilistic & Weighting sum & & & \\
\hline 20 & 0.00 & 0.44 & 0.53 & 0.18 & 0.00 & $0.00(w=0.49)$ & 0.87 & 0.87 & 1.71 \\
\hline 30 & 0.13 & 0.50 & 0.49 & 0.19 & 0.15 & $0.11(w=0.51)$ & 0.79 & 0.93 & 1.57 \\
\hline 40 & 0.19 & 0.49 & 0.54 & 0.20 & 0.18 & $0.18(w=0.49)$ & 0.78 & 0.87 & 1.28 \\
\hline 50 & 0.26 & 0.56 & 0.54 & 0.27 & 0.27 & $0.24(w=0.52)$ & 0.81 & 0.85 & 1.15 \\
\hline 90 & 0.39 & 0.55 & 0.54 & 0.40 & 0.41 & $0.39(w=0.50)$ & 0.67 & 0.67 & 0.82 \\
\hline
\end{tabular}

person is identified as a registered person with the shortest Euclidean distance. In verification, the system calculates the Euclidean distance $D^{r}$ for target person, if the Euclidean distance is shorter than a threshold, the system accepts the walking person.

We performed authentication for 20, 30, 40, 50, and 90 volunteers as shown in Table 3 . Tables 4,5 , and 6 show a comparison of authentication results for each number of subjects. From these results, we can see that the weighted sum operator had lower FRR and FAR than the other operators. The probabilistic sum and the mean operators obtained lower EER than the others. The mean, probabilistic sum, and weighting sum operators had similar FRR, FAR, and EER. Figure 14 shows FRR and FAR of the mean operator for 20 and 90 subjects with the threshold $[0,1]$. The authentication performance of any operator decreases with the increment of the number. Figure 15 shows the FRR and EER of with the weighting parameter $[0,1]$. From this figure, we can see that the operator obtained the lowest FRR and EER when the weighting parameter was almost 0.5 . From this fact, we consider that the mean operator was an optimal operator for sole pressure-based biometrics. Moreover, all combination operators achieved lower error rates than the results using only right or left sole pressure data.

\section{Discussion}

We compared the proposed method with the biometrics based on walking. The proposed method achieved $12.7 \%$ FRR in 50 subjects. Considering past recognition systems by walking data, [18] achieved $85 \%$ recognition rate (15\% FRR) in 10 subjects. The comparison method has authenticated person based on image matching between acquired footprints and template. Reference [19] achieved 4.3\% FAR and $65.1 \%$ FRR in 8 subjects. The method used center of pressures trajectory of one foot for authentication. The center of pressure is modeled by Hidden Markov Model. And [8] did $0.14 \%$ FAR and $1.36 \%$ FRR in 11 subjects. The method used both footprint and center of pressure trajectory. The method calculated two authentication scores from footprint based on template matching and center of pressure based on Hidden Markov Model. Then a LM learning method weighed each score, and the method calculated authentication score by weighted sum operation. Our method achieved the lowest FRR among these methods. Thus, our method aided by fuzzy logic would be a stepping stone future footprint biometric system.

From our experimental results, there was no great difference between the authentication performances by using 
TABLE 6: EERs in verification of combination operators.

\begin{tabular}{|c|c|c|c|c|c|c|c|c|c|}
\hline \multirow{3}{*}{ Volunteers (person) } & \multicolumn{9}{|c|}{$\operatorname{EER}(\%)$} \\
\hline & \multicolumn{6}{|c|}{ Combination operators } & \multirow{2}{*}{$\mu_{R}^{r}(16)$} & \multirow{2}{*}{$\mu_{L}^{r}$} & \multirow{2}{*}{ Euclidean distance } \\
\hline & Mean (17) & Minimum & Maximum & Product & Probabilistic & Weighting sum & & & \\
\hline 20 & 3.27 & 5.57 & 4.12 & 3.33 & 2.52 & $2.52(w=0.49)$ & 8.09 & 7.37 & 17.5 \\
\hline 30 & 3.33 & 6.65 & 4.44 & 3.33 & 3.29 & $3.33(w=0.51)$ & 9.58 & 8.44 & 19.4 \\
\hline 40 & 4.17 & 7.12 & 5.01 & 4.57 & 3.76 & $4.15(w=0.49)$ & 9.69 & 9.35 & 21.8 \\
\hline 50 & 5.25 & 8.65 & 6.36 & 5.66 & 5.09 & $5.11(w=0.52)$ & 10.60 & 11.92 & 23.3 \\
\hline 90 & 8.78 & 11.33 & 10.30 & 9.03 & 8.90 & $8.78(w=0.50)$ & 13.49 & 14.97 & 29.2 \\
\hline
\end{tabular}

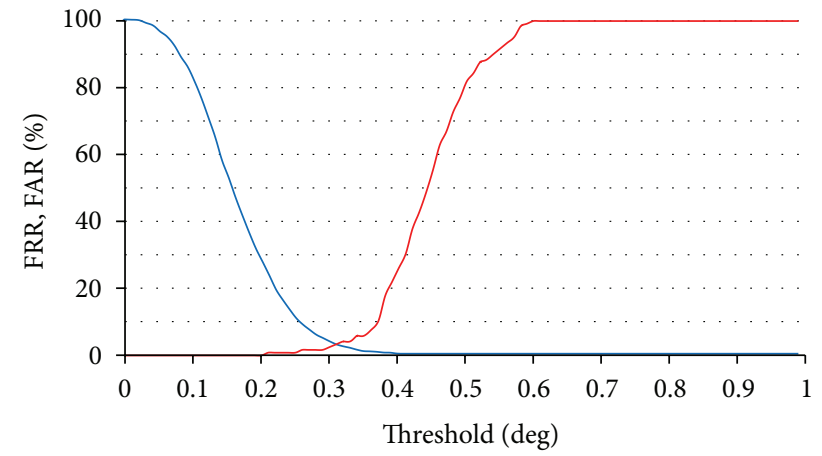

(a) 20 volunteers

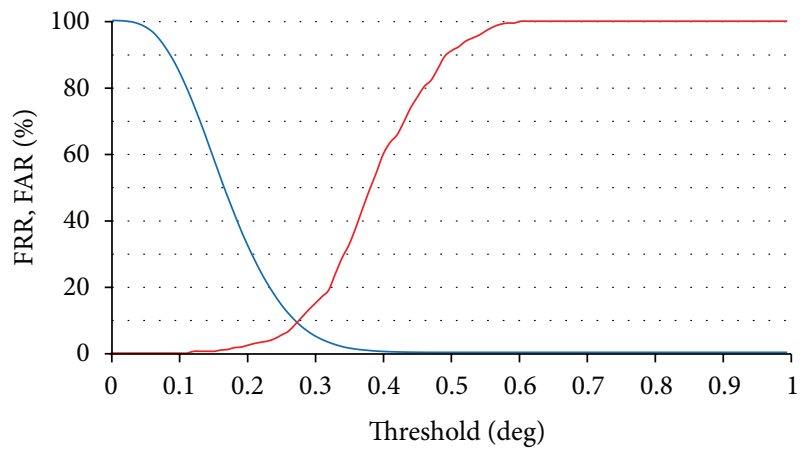

(b) 90 volunteers

FIGURE 14: FRR and FAR of verification with threshold change on mean operator.

right sole $\left(\mu_{R}^{r}\left(X_{R}^{S}\right)\right)$ and left sole $\left(\mu_{L}^{r}\left(X_{L}^{S}\right)\right)$. Furthermore, our method obtained the best authentication accuracy when the weighing parameter was almost 0.5 . However, the authentication performances were improved by using the combined fuzzy degree. From this fact, we consider that the personal authentication method based on the combined fuzzy degree can be used for other biometrics authentication system to improve its performance.

In all operators, authentication performances were deteriorated with increasing the number of subjects. Our method totally employed seventy-eight features from both sole pressures. However, the normalized CSPs were extracted from CSP trajectory, and the trajectory explains a pattern of dynamics of weight shift. Thus, we treat the nine normalized CSPs as one feature. In the same way, we consider that the normalized HSPs and areas are one feature in each. Thus,

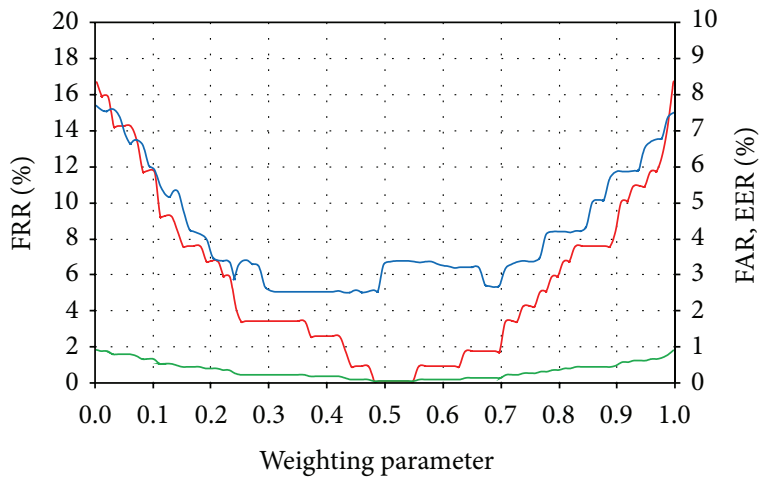

(a) 20 volunteers

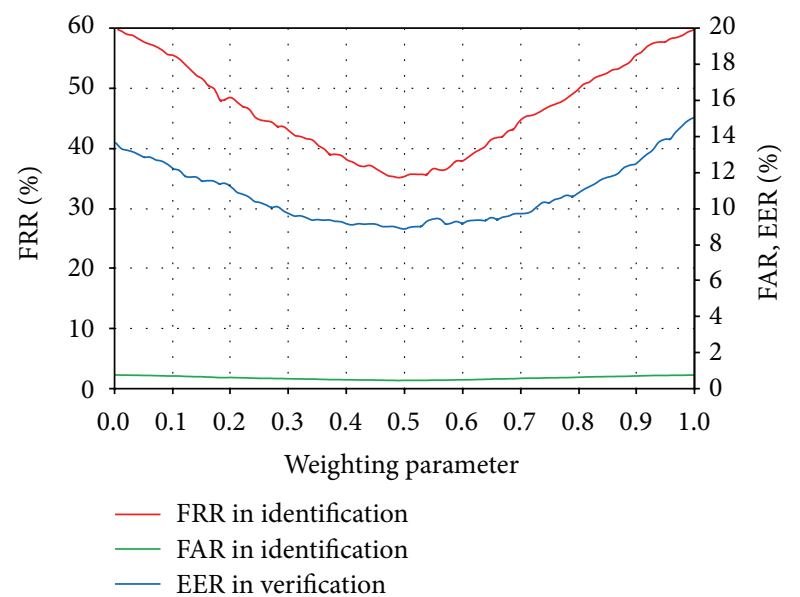

(b) 90 volunteers

FIGURE 15: Authentication performances on weighting sum operator.

thirty features were substantially used for authentication. By the limit of dimensionality, we consider that authentication performances were deteriorated in over thirty volunteers.

\section{Conclusions}

We have proposed a biometric personal authentication system based on fuzzy logic. We employed right and left sole pressure distributions during walking. A mat-type load distribution sensor acquired the sole pressure data. In authentication, the biometrics system extracted thirty-nine features 
from each sole pressure data, and it described fuzzy ifthen rules with statistically determined fuzzy membership functions in learning process for each feature. A fuzzy degree of both sole pressure data was calculated by combination operators. It was evaluated in the personal authentication. In identification, the system calculated the combined fuzzy degree for every registered person, and it identified walking person as a registered person with the highest fuzzy degree. In verification, we calculated the fuzzy degree for the target person. When the fuzzy degree is higher than a threshold, we verified the walking person as the target person. In the experiments, we identified $0.0 \%$ in FRR and $0.0 \%$ in FAR, and we verified $2.52 \%$ in EER by the weighted sum operator on 20 subjects. We did $35.0 \%$ in FRR and $0.39 \%$ in FAR, and we verified $8.85 \%$ in EER in 90 subjects. Thus, this system achieved good performance on small number of subjects. In our experiment, we consider the six combination operators. The mean operator obtained the lower error rates in authentication. We consider the mean operator as an optimal operator for combining left and right sole pressures for biometric security. In comparison with the other methods by walking data, our method achieved the lowest FRR.

Health monitoring for walking condition is also an important research for human healthcare. In the feature, we will improve the performance by optimizing the fuzzy membership functions and add other biometric features to the method and authenticate person by multimodal approach.

\section{References}

[1] K. Ito, H. Nakajima, K. Kobayashi, T. Aoki, and T. Higuchi, "A fingerprint matching algorithm using phase-only correlation," IEICE Transactions on Fundamentals of Electronics, Communications and Computer Sciences, vol. E87-A, no. 3, pp. 682-691, 2004.

[2] X. Chen, J. Tian, and X. Yang, "A new algorithm for distorted fingerprints matching based on normalized fuzzy similarity measure," IEEE Transactions on Image Processing, vol. 15, no. 3, pp. 767-776, 2006.

[3] A. Kholmatov and B. Yanikoglu, "Identity authentication using improved online signature verification method," Pattern Recognition Letters, vol. 26, no. 15, pp. 2400-2408, 2005.

[4] D. A. Reynolds and R. C. Rose, "Robust text-independent speaker identification using Gaussian mixture speaker models," IEEE Transactions on Speech and Audio Processing, vol. 3, no. 1, pp. 72-83, 1995.

[5] D. K. Wagg and M. S. Nixon, "On automated model-based extraction and analysis of gait," in Proceedings of the 6th IEEE International Conference on Automatic Face and Gesture Recognition (FGR '04), pp. 11-16, Seoul, Korea, May 2004.

[6] L. Wang, H. Ning, T. Tan, and W. Hu, "Fusion of static and dynamic body biometrics for gait recognition," IEEE Transactions on Circuits and Systems for Video Technology, vol. 14, no. 2, pp. 149-158, 2004.

[7] J. Mäntyjärvi, M. Lindholm, E. Vildjiounaite, S.-M. Mäkelä, and H. Ailisto, "Identifying users of portable devices from gait pattern with accelerometers," in Proceedings of the IEEE International Conference on Acoustics, Speech, and Signal Processing (ICASSP '05), pp. II973-II976, March 2005.
[8] J.-W. Jung, Z. Bien, and T. Sato, "Person recognition method using sequential walking footprints via overlapped foot shape and center-of-pressure trajectory," IEICE Transactions on Fundamentals of Electronics, Communications and Computer Sciences, vol. E87-A, no. 6, pp. 1393-1400, 2004.

[9] G. Qian, J. Zhang, and A. Kidane, "People identidication using gait via floor pressure sensing and analysis," in Proceedings of the 3rd European Conference on Smart Sensing and Context, pp. 83-98, Zurich, Switzerland, October 2008.

[10] M. D. Addlesee, A. Jones, F. Livesey, and F. Samaria, "The ORL active floor," IEEE Personal Communications, vol. 4, no. 5, pp. 35-41, 1997.

[11] R. J. Orr and G. D. Abowd, "The smart floor: a mechanism for natural user identification and tracking," in Proceedings of the Conference on Human Factors in Computing Systems, The Hague, The Netherlands, April 2000.

[12] T. Yamakawa, K. Taniguchi, T. Momen, S. Kobashi, K. Kondo, and Y. Hata, "Biometric personal identification based on foot pressure change," in Proceedings of the International Conference on Soft Computing and Human Sciences, pp. 83-86, Fukuoka, Japan, 2007.

[13] T. Takeda, K. Taniguchi, K. Asari, K. Kuramoto, S. Kobashi, and Y. Hata, "Biometric personal authentication by one step foot pressure distribution change by load distribution sensor," in Proceedings of the IEEE International Conference on Fuzzy Systems, pp. 906-910, August 2009.

[14] T. Takeda, K. Taniguchi, K. Asari, K. Kuramoto, S. Kobashi, and Y. Hata, "Biometric personal identification by dinamics of sole pressure at walking," in Proceedings of the World Automation Congress (WAC '10), Kobe, Japan, September 2010.

[15] V. Conti, C. Militello, F. Sorbello, and S. Vitabile, "A frequencybased approach for features fusion in fingerprint and iris multimodal biometric identification systems," IEEE Transactions on Systems, Man and Cybernetics C, vol. 40, no. 4, pp. 384-395, 2010.

[16] A. K. Jain, S. Prabhakar, and S. Chen, "Combining multiple matchers for a high security fingerprint verification system," Pattern Recognition Letters, vol. 20, no. 11-13, pp. 1371-1379, 1999.

[17] J. Kittler, J. Matas, K. Jonsson, and M. U. R. Sánchez, “Combining evidence in personal identity verification systems," Pattern Recognition Letters, vol. 18, no. 9, pp. 845-852, 1997.

[18] K. Nakajima, Y. Mizukami, K. Tanaka, and T. Tamura, "Footprint-based personal recognition," IEEE Transactions on Biomedical Engineering, vol. 47, no. 11, pp. 1534-1537, 2000.

[19] J.-W. Jung, Z. Bien, S.-W. Lee, and T. Sato, "Dynamic-footprint based person identification using mat-type pressure sensor," in Proceedings of the 25th Annual International Conference of the IEEE Engineering in Medicine and Biology Society (EMBC '03), vol. 3, pp. 2937-2940, Cancun, Mexico, September 2003. 

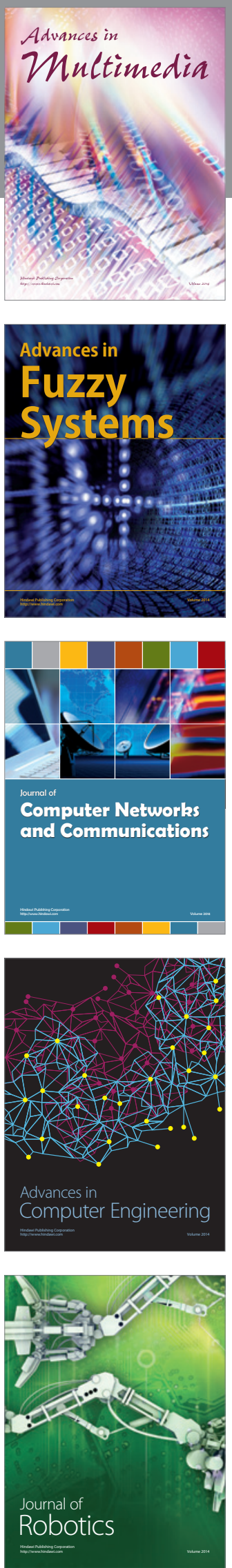

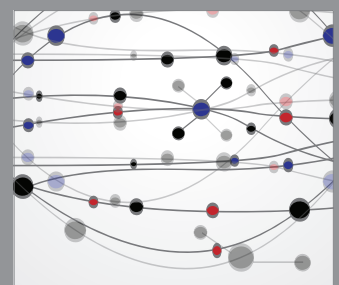

The Scientific World Journal
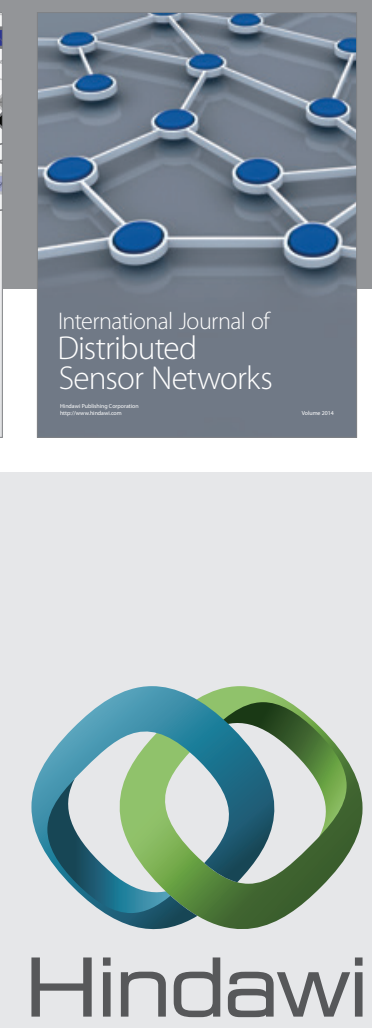

Submit your manuscripts at

http://www.hindawi.com
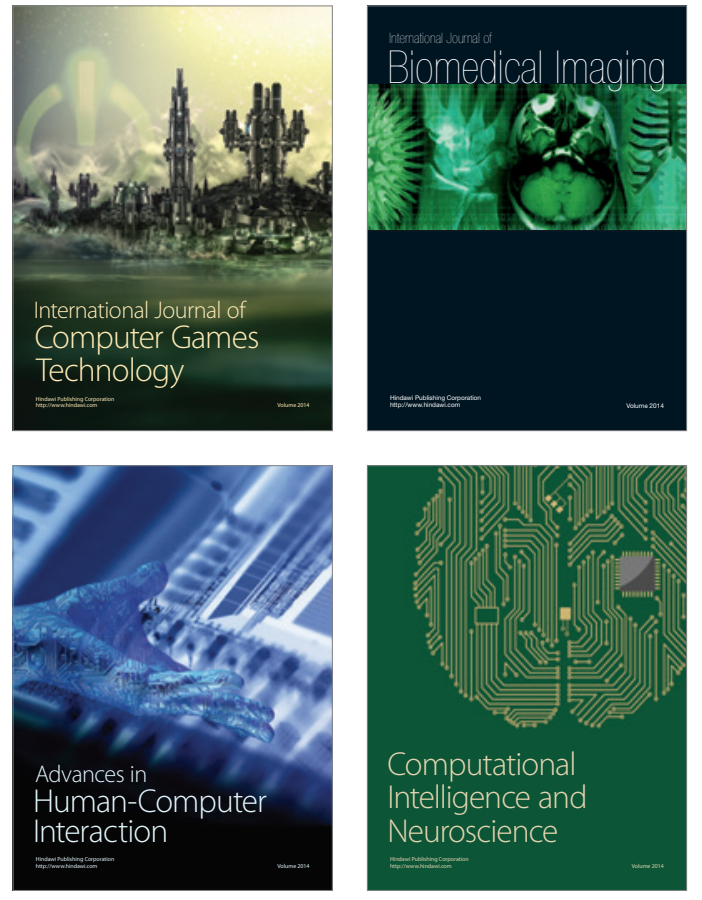
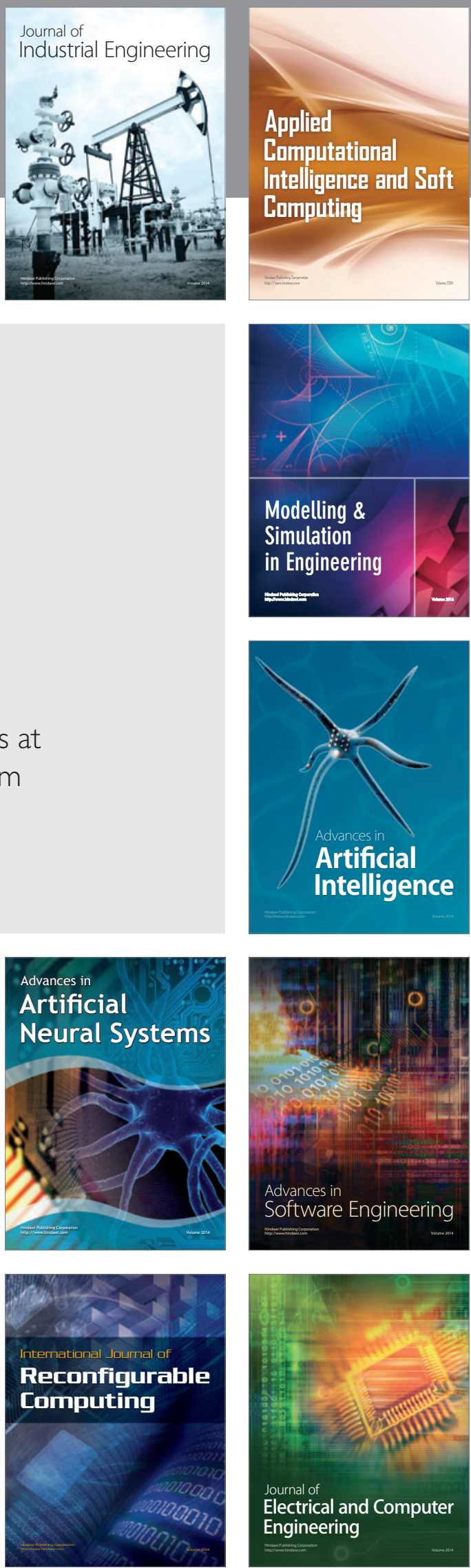